\title{
A DECOMPOSITION RELATIVE TO CONVEX SETS
}

M. Z. NASHED

1. The purpose of this note is to prove a decomposition theorem which asserts that, given a closed convex set $C$ in a Hilbert space $H$, each element $u \in H$ can be uniquely decomposed as the sum of an element $x \in H$ and the closest-point projection of $x$ on $C$. Moreover, $x$ depends continuously on $u$ and can be determined by an iterative procedure. We also prove a theorem on the solution by iteration of monotone operator equations.

2. In what follows, $H$ will denote a real or a complex Hilbert space with inner product $\langle u, v\rangle$. Recall that an operator $T: D \rightarrow H$ is called monotonic on a domain $D \subset H$ if for all $u, v \in D, \operatorname{Re}\langle u-v, T u-T v\rangle \geqq 0$; $T$ is called strongly monotonic if the zero in this inequality is replaced by $m\|u-v\|^{2}$, where $m$ is some positive number. For the original contributions to the theory of monotone operators as well as for a survey of related literature and applications to differential and integral equations, see for instance [1], [2], [3], [5], [6]. Let $T: H \rightarrow H$ be a continuous monotone operator. Then the equation $x+T x=y$ has a unique solution for each $y \in H$ and the solution depends continuously on $y$ (see [3]). The monotonicity hypothesis can be weakened. The following theorem is due to Browder [1, Theorem 4].

Theorem 1. Let $G: H \rightarrow H$ be a continuous mapping such that for all $u, v \in H$,

$$
\operatorname{Re}\langle G u-G v, u-v\rangle \geqq m(\max \{\|u\|,\|v\|\})\|u-v\|^{2},
$$

where $m(t)$ is a positive nonincreasing function of $t$ such that

$$
\int_{1}^{\infty} m(t) d t=+\infty
$$

Then $G$ is one-to-one, onto, and its inverse is continuous.

We now prove

THEOREM 2. Let $Q$ be an operator (not necessarily linear) from $H$ into $H$ with the property that $Q u=\theta$ if and only if $u=\theta$. Let $G: H \rightarrow H$ satisfy the conditions

$$
\|Q G u-Q G v\| \leqq M(\max \{\|u\|,\|v\|\})\|u-v\|
$$

Presented to the Society, February 13, 1967 under the title $A$ decomposition relaive to convex sets; received by the editors February 15, 1967. 
and

$$
\operatorname{Re}\langle Q G u-Q G v, u-v\rangle \geqq m(\max \{\|u\|,\|v\|\})\|u-v\|^{2},
$$

where $m(t)$ is a positive nonincreasing function such that (2) and (5) hold and $M(t)$ is a positive nondecreasing function. Let

$$
r=2 \sup \{t: \operatorname{tm}(t) \leqq\|Q G \theta\|\}<\infty,
$$

let $\beta$ be any number in the range ${ }^{1}$

$$
0<\beta<m^{2}(r) / M^{2}(r),
$$

and let $a$ and $b$ be the (positive) roots of the equation

$$
M^{2}(r) \alpha^{2}-2 m(r) \alpha+\beta=0 .
$$

Then starting with the initial approximation $x_{0}=\theta$, the iterative process

$$
x_{n+1}=x_{n}-\alpha_{n} Q G x_{n}, \quad n=0,1, \cdots,
$$

where $a \leqq \alpha_{n} \leqq b$, converges to the unique solution $x^{*}$ of $G x=\theta$ and

$$
\left\|x_{n}-x^{*}\right\| \leqq \frac{1}{2}(1-\beta)^{n / 2} r .
$$

Proof. The existence and uniqueness of the solution follow from Theorem 1.

From Schwarz's inequality we get

$$
\|Q G x-Q G \theta\|\|x\| \geqq \operatorname{Re}\langle x, Q G x-Q G \theta\rangle \geqq m(\|x\|)\|x\|^{2}
$$

and hence

$$
\|Q G x\| \geqq\|Q G x-Q G \theta\|-\|Q G \theta\| \geqq m(\|x\|)\|x\|-\|Q G \theta\| .
$$

Thus

$$
m\left(\left\|x^{*}\right\|\right)\left\|x^{*}\right\| \leqq\|Q G \theta\| .
$$

Let $r$ be as defined in (5). Then $\left\|x^{*}\right\| \leqq r / 2$. Now consider the iteration (6). Taking $x_{0}=\theta$ we get $\left\|x^{*}-x_{0}\right\| \leqq r / 2$. We shall show by induction that for $\alpha_{n}$ in a certain range, all the iterates will lie in $\|x-x *\|$ $\leqq r / 2$. Assume that $x_{1}, \cdots, x_{n}$ satisfy $\left\|x-x^{*}\right\| \leqq r / 2$. Then

$$
\begin{aligned}
\left\|x_{n+1}-x^{*}\right\|^{2} \leqq & {\left[1-2 \alpha_{n} m\left(\max \left\{\left\|x_{n}\right\|,\left\|x^{*}\right\|\right\}\right)\right.} \\
& \left.\quad+\alpha_{n}^{2} M^{2}\left(\max \left\{\left\|x_{n}\right\|,\left\|x^{*}\right\|\right\}\right)\right]\left\|x_{n}-x^{*}\right\|^{2} \\
\leqq & {\left[1-2 \alpha_{n} m(r)+\alpha_{n}^{2} M^{2}(r)\right]\left\|x_{n}-x^{*}\right\|^{2} . }
\end{aligned}
$$

Thus if there exists a $\beta, 0<\beta<1$, such that

${ }^{1}$ Note that (3) and (4) imply $m(t) \leqq M(t)$; hence $\beta<1$. 


$$
1-2 \alpha_{n} m(r)+\alpha_{n}^{2} M^{2}(r) \leqq 1-\beta
$$

for all $n$, then $x_{n+1} \in S(\theta, r)$ and

$$
\left\|x_{n+1}-x^{*}\right\|^{2} \leqq(1-\beta)^{n+1}\left\|x_{0}-x^{*}\right\|^{2} \leqq(1-\beta)^{n+1} r^{2} / 4 .
$$

Hence by induction $x_{n} \in S\left(x^{*}, r / 2\right)$ for all $n$. To complete the proof, note that (7) holds under the assumptions of the theorem.

REMARK 1. Results analogous to Theorems 1 and 2 may be stated for equations of the type

$$
x+\lambda T x=y
$$

where $T$ is a mapping from $H$ into $H, y$ is a fixed element in $H$ and $\lambda$ is a scalar. For example, if we require that $\lambda T$ be monotonic and continuous, then the operator $G$ defined by $G x=x+\lambda T x-y$ is continuous and strongly monotonic since for any $u, v \in H$,

$$
\operatorname{Re}\langle G u-G v, u-v\rangle \geqq\|u-v\|^{2} .
$$

Under these conditions, (8) has a unique solution for each $y$.

3. A decomposition theorem. Let $C$ be a closed convex set in a Hilbert space $H$ and let $P$ denote the "projection" operator on $C$. This is the operator which assigns to each $x \in H$ its closest point $P x \in C$, i.e.,

$$
\|P x-x\|=\inf \{\|y-x\|: y \in C\} .
$$

It is well known that $P$ exists and is single-valued (e.g., [4]). $P$ is also called the closest-point map on $C$. We shall show in Theorem 3 that each $u \in H$ can be uniquely decomposed as the sum of an element $x \in H$ and its projection $P x \in C$; moreover $x$ depends continuously on $u$ and can be determined by an iterative process. We first prove the following.

Lemma. The closest-point map $P$ on a closed convex set $C$ in a Hilbert space $H$ is monotonic. $P$ is not strongly monotonic if $C \not H$.

Proof. It is easy to show that a point $z \in C$ is the closest point to $y \notin C$ if and only if

$$
\operatorname{Re}\langle x-z, z-y\rangle \geqq 0 \text { for all } x \in C .
$$

Note that $x_{t}=t x+(1-t) z \in C$ for $0 \leqq t \leqq 1$ and thus $\left\|y-x_{t}\right\|^{2}-\|y-z\|^{2}$ $\geqq 0$. Expansion of the left side of this inequality leads to (9). Thus for any $x, y \in H$ we have $\operatorname{Re}\langle x-P x, P x-P y\rangle \geqq 0$ and $\operatorname{Re}\langle P y-y, P x-P y\rangle$ $\geqq 0$. Adding terms we get $\operatorname{Re}\langle x-y-(P x-P y), P x-P y\rangle \geqq 0$. Thus

$$
\operatorname{Re}\langle x-y, P x-P y\rangle \geqq\|P x-P y\|^{2} \geqq 0,
$$


which proves the monotonicity of $P$.

To prove the second part of the lemma, suppose $C \not \neq H$ and choose $x \notin C$. Let $y=2 x-P x$. Then for all $z \in C$,

$$
\|x-P x\| \leqq\left\|x-\frac{1}{2}(P x+z)\right\|
$$

or

$$
\|y-P x\| \leqq\|y-z\|
$$

Hence $P y=P x$ and

$$
\operatorname{Re}\langle x-y, P x-P y\rangle=0 .
$$

Thus, if $P$ is strongly monotonic,

$$
\operatorname{Re}\langle x-y, P x-P y\rangle \geqq m\|x-y\|^{2}, \quad m>0,
$$

we must have $y=x$. This implies $x=P x$. But this is a contradiction since $x \notin C$.

Theorem 3. Let $C$ be a closed convex set in $H$. For each $u \in H$ there exists a unique $x \in H$ such that $u-x$ is the point in $C$ closest to $x$, and $x$ depends continuously on $u$. Furthermore, if $a$ and $b$ are the roots of the equation $\alpha^{2}-\alpha+q=0$, where $0<q<1 / 2$, then the sequence

$$
x_{n+1}=\left(1-\alpha_{n}\right) x_{n}+\alpha_{n}\left(u-P x_{n}\right), \quad n=0,1, \cdots,
$$

where $a \leqq \alpha_{n} \leqq b$, converges to $x$ starting from an arbitrary initial $a p$ proximation $x_{0} \in H$.

Proof. Consider the equation $x+P x=u$, where $P$ is the closestpoint map on $C$ and $u \in H$. By the lemma, $P$ is monotonic on $H$. Furthermore, we know that $P$ is continuous and distance shrinking:

$$
\left\|P x_{1}-P x_{2}\right\| \leqq\left\|x_{1}-x_{2}\right\| \text { for any } x_{1}, x_{2} \in H \text {. }
$$

(This also follows from inequality (10).) Thus the first part of the theorem follows from Theorem 1 where $G=I+P$ and $m=1$. This part of the theorem also follows directly from a simple argument based on the contraction principle applied to the mapping $T_{\lambda}=(1-\lambda) I$ $+\lambda(u-P)$, where $0<\lambda<1 / 2$. Indeed for $\lambda \neq 0$, every fixed point of $T_{\lambda}$ is a solution of the equation $x+P x=u$ and conversely. An easy computation shows that $T_{\lambda}$ is a strict contraction for $0<\lambda<1 / 2$. The second part follows as a special case of Theorem 2 by noting that in this case $G x=x+P x-u, Q=I, m=1$, and $M \leqq 2$.

REMARK 2. In the iteration (12), we have tacitly assumed that we can constructively "project" points on $C$. Since this can be usually done only for some classes of convex sets, our procedure is not com- 
pletely constructive. See [7], [8] for some aspects of approximating the closest-point map.

REMARK 3. It is known (e.g., [4]) that the closest-point map exists and is single-valued for every closed convex set in a strictly convex normed linear space if and only if the space is reflexive. In view of this, it may be interesting to determine whether one can obtain a characterization of strictly convex reflexive normed linear spaces in terms of the convergence property stated in Theorem 3 .

Note that it is possible to give a simple expression for the decomposition valid in any strictly convex normed space, namely, $x=u-P(u / 2)$. To show that this is the desired $x$, one need only show that $P(x)=P(u / 2)$, which result is implicit in the proof of the second part of the Lemma.

\section{REFERENCES}

1. F. E. Browder, On the solvability of nonlinear functional equations, Duke Math. J. 30 (1963), 557-566.

2. - Nonlinear boundary value problems, pp. 24-49, Proc. Sympos. Appl. Math. Vol. 17, Amer. Math. Soc., Providence, R. I., 1965.

3. C. L. Dolph and G. J. Minty, "On nonlinear integral equations of the Hammerstein type" in Nonlinear integral equations, edited by P. M. Anselone, Univ. of Wisconsin Press, Madison, 1964.

4. R. R. Phelps, Uniqueness of the Hahn-Banach extension and unique best approximation, Trans. Amer. Math. Soc. 95 (1960), 238-255.

5. E. M. Zarantonello, Solving functional equations by contractive averaging, Tech. Summary Rep. 160, Math. Research Ctr., U. S. Army, 1960.

6. - The closure of the numerical range contains the spectrum, Pacific J. Math. 22 (1967).

7. F. R. Deutsch and P. H. Maserick, Applications of the Hahn-Banach theorem in approximation theory, SIAM Rev. 9 (1967), 513-530.

8. R. E. Holmes, Approximating best approximations, Nieuw Arch. Wisk. (3) 14 (1966), 106-113.

Georgia Institute of Technology 